\title{
Pharyngeal Stripping Wave Present but Diminished
}

National Cancer Institute

\section{Source}

National Cancer Institute. Pharyngeal Stripping Wave Present but Diminished. NCI

Thesaurus. Code C127312.

A finding of a pharyngeal stripping wave present but diminished. 